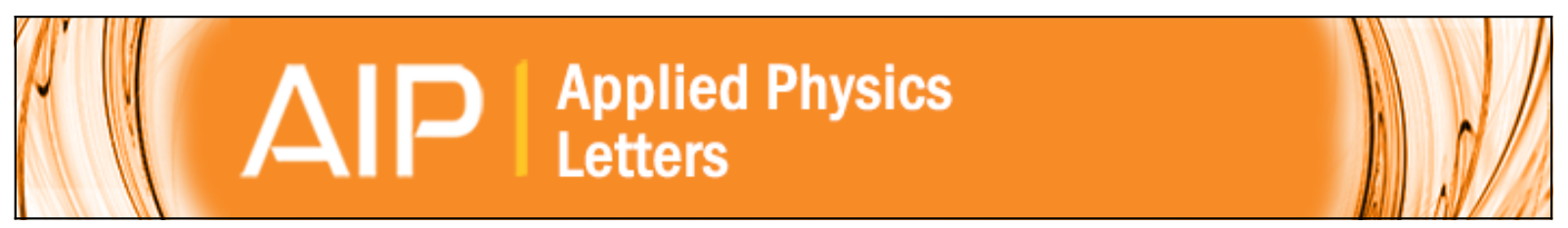

\title{
Strain-driven growth of GaAs(111) quantum dots with low fine structure splitting
}

Christopher D. Yerino, Paul J. Simmonds, Baolai Liang, Daehwan Jung, Christian Schneider, Sebastian

Unsleber, Minh Vo, Diana L. Huffaker, Sven Höfling, Martin Kamp, and Minjoo Larry Lee

Citation: Applied Physics Letters 105, 251901 (2014); doi: 10.1063/1.4904944

View online: http://dx.doi.org/10.1063/1.4904944

View Table of Contents: http://scitation.aip.org/content/aip/journal/apl/105/25?ver=pdfcov

Published by the AIP Publishing

\section{Articles you may be interested in}

Tensile GaAs(111) quantum dashes with tunable luminescence below the bulk bandgap

Appl. Phys. Lett. 105, 071912 (2014); 10.1063/1.4893747

Highly uniform and strain-free GaAs quantum dots fabricated by filling of self-assembled nanoholes Appl. Phys. Lett. 94, 183113 (2009); 10.1063/1.3133338

Effects of In x Ga 1 - x As matrix layer on InAs quantum dot formation and their emission wavelength

J. Appl. Phys. 100, 033109 (2006); 10.1063/1.2220477

Spatial correlation-anticorrelation in strain-driven self-assembled InGaAs quantum dots

Appl. Phys. Lett. 85, 1356 (2004); 10.1063/1.1784526

Effect of growth temperature on luminescence and structure of self-assembled InAIAs/AIGaAs quantum dots J. Appl. Phys. 90, 2048 (2001); 10.1063/1.1388021

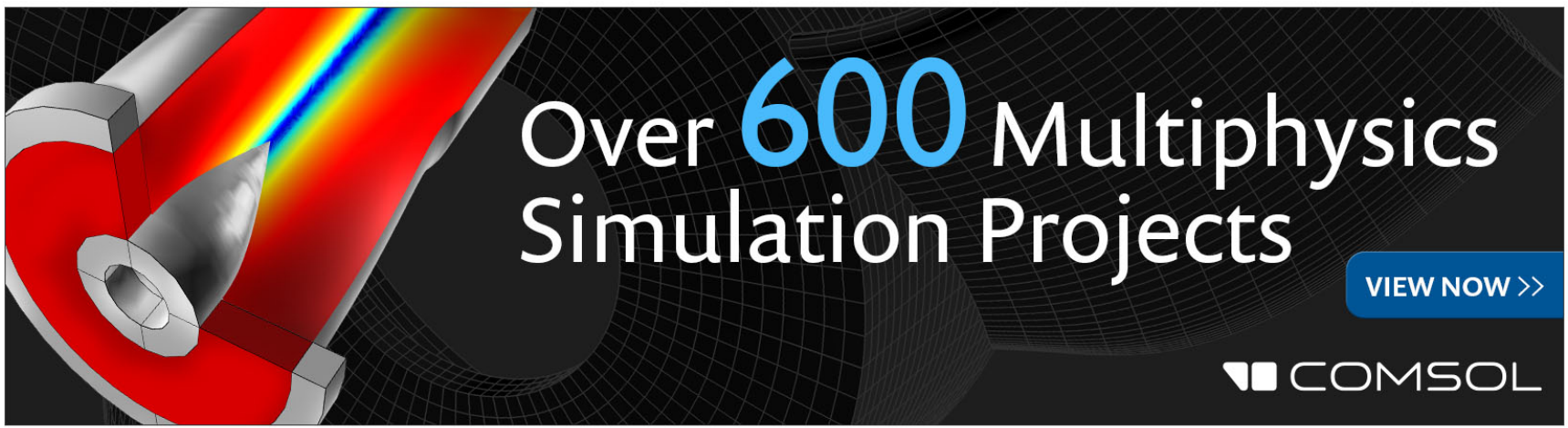




\title{
Strain-driven growth of $\mathrm{GaAs}(111)$ quantum dots with low fine structure splitting
}

\author{
Christopher D. Yerino, ${ }^{1}$ Paul J. Simmonds, ${ }^{2}$ Baolai Liang, ${ }^{3}$ Daehwan Jung, ${ }^{1}$ \\ Christian Schneider, ${ }^{4}$ Sebastian Unsleber, ${ }^{4}$ Minh Vo, ${ }^{4}$ Diana L. Huffaker, ${ }^{3}$ Sven Höfling, ${ }^{4,5}$ \\ Martin Kamp, ${ }^{4}$ and Minjoo Larry Lee ${ }^{1, a)}$ \\ ${ }^{1}$ Department of Electrical Engineering, Yale University, New Haven, Connecticut 06520, USA \\ ${ }^{2}$ Departments of Physics and Materials Science and Engineering, Boise State University, Boise, Idaho 83725, \\ USA \\ ${ }^{3}$ California NanoSystems Institute and Department of Electrical Engineering, University of California, Los \\ Angeles, California 90095, USA \\ ${ }^{4}$ Technische Physik, Physikalisches Institut and Wilhelm Conrad Röntgen-Research Center for Complex \\ Material Systems, Universität Würzburg, Am Hubland, D-97074 Würzburg, Germany \\ ${ }^{5}$ SUPA, School of Physics and Astronomy, University of St. Andrews, St. Andrews, KY16 9SS, \\ United Kingdom
}

(Received 24 October 2014; accepted 11 December 2014; published online 22 December 2014)

Symmetric quantum dots (QDs) on (111)-oriented surfaces are promising candidates for generating polarization-entangled photons due to their low excitonic fine structure splitting (FSS). However, (111) QDs are difficult to grow. The conventional use of compressive strain to drive QD selfassembly fails to form 3D nanostructures on (111) surfaces. Instead, we demonstrate that (111) QDs self-assemble under tensile strain by growing GaAs QDs on an InP(111)A substrate. Tensile GaAs self-assembly produces a low density of QDs with a symmetric triangular morphology. Coherent, tensile QDs are observed without dislocations, and the QDs luminescence at room temperature. Single QD measurements reveal low FSS with a median value of $7.6 \mu \mathrm{eV}$, due to the high symmetry of the (111) QDs. Tensile self-assembly thus offers a simple route to symmetric (111) QDs for entangled photon emitters. (C) 2014 AIP Publishing LLC.

[http://dx.doi.org/10.1063/1.4904944]

Quantum dots (QDs) have great potential for entangled photon sources in quantum information, ${ }^{1}$ since they can generate pairs of polarization-entangled photons via a biexciton-exciton cascade. ${ }^{2,3}$ The entangled two-photon states are energetically indistinguishable, provided that the exciton levels have negligible fine structure splitting (FSS). ${ }^{4}$ However, non-zero FSS often occurs in QDs due to asymmetric QD confinement potentials. This asymmetry typically arises for QDs grown on (001) surfaces due to their in-plane piezoelectric field and their commonly asymmetric shape. ${ }^{4}$ Low FSS has been achieved in (001) QDs, but it typically requires postgrowth adjustments, such as careful selection of low FSS QDs from a wide size distribution, or external manipulation using magnetic fields. ${ }^{4,5}$ In contrast, QDs grown on (111) surfaces have been proposed as ideal sources of entangled photon pairs, since their piezoelectric field naturally lies perpendicular to the surface, preserving the electronic symmetry. ${ }^{6,7}$

(111) QDs have nonetheless proven extremely challenging to grow. Attempts to form compressively strained QDs (e.g., InAs on GaAs) on (111) surfaces by the conventional Stranski-Krastanov (S-K) growth mode do not produce 3D nanostructures. Instead of assembling QDs, the compressive strain relaxes plastically to form dense misfit dislocation arrays. ${ }^{8,9}$ The failure of S-K growth on (111) surfaces has driven the development of alternative (111) QD growth techniques including droplet epitaxy ${ }^{10-12}$ and growth on patterned substrates. ${ }^{13,14}$ In droplet epitaxy, metal droplets

\footnotetext{
${ }^{\text {a)}}$ Electronic mail: minjoo.lee@yale.edu
}

deposited onto the substrate are crystallized under arsenic vapor to form III-V semiconductor QDs. QD crystallization requires low temperature to preserve the $3 \mathrm{D}$ morphology, ${ }^{15}$ but the low temperature growth also creates arsenic-related defects that require annealing to mitigate and are challenging to eliminate completely. ${ }^{16}$ Nonetheless, progress in droplet epitaxy has led to (111) QDs with narrow linewidths and low FSS. ${ }^{10,11}$ Growth within pyramidal etch-pits on (111) substrates offers an alternative, whereby preferential growth at the bottom of each pit produces 3D QDs. However, the pitgeometry hinders light extraction from the QDs, ${ }^{13}$ and large etch-pits are difficult to integrate into photonic resonators for high photon emission rates. ${ }^{17}$ Despite these challenges, the etch-pit method has demonstrated low FSS and a high yield of entangled photon emitters through elaborate substrate processing. ${ }^{13}$

In this work, we re-visit the challenge of strain-driven QD growth on (111) surfaces as a simpler, single-step route to high symmetry QDs. We have recently shown that tensile strain is effective at driving the self-assembly of dislocationfree nanostructures on (111) surfaces. ${ }^{18-20}$ Compared to compressive strain, tensile strain is advantageous for (111) growth since the latter produces a higher energetic barrier to dislocation nucleation. ${ }^{19}$ Thus, the epitaxial strain can more efficiently drive the self-assembly of 3D nanostructures. Based on this principle, tensile strain has been used to selfassemble dislocation-free nanostructures in different material systems, including GaAs on $\operatorname{In}_{0.52} \mathrm{Al}_{0.48} \mathrm{As}(111) \mathrm{B},{ }^{18} \mathrm{GaP}$ on $\operatorname{GaAs}(111) \mathrm{A},{ }^{20}$ and $\mathrm{Si}$ on $\mathrm{Ge}(111) .{ }^{21}$ However, the growth 
of symmetric QDs in an optically active material system has not yet been established using this technique. Here we demonstrate the use of tensile self-assembly to produce highsymmetry GaAs(111)A QDs on an InP substrate. We report triangular-shaped GaAs(111)A QDs that are coherently strained and exhibit photoluminescence (PL) up to room temperature. Due to the high symmetry produced by the (111) surface, the QDs exhibit low FSS, a key requirement for the generation of polarization-entangled photons.

The tensile $\operatorname{GaAs}(111) \mathrm{A}$ QDs are grown within $\mathrm{In}_{0.52} \mathrm{Al}_{0.48} \mathrm{As}$ barriers (InAlAs) on semi-insulating, nominally exact $\operatorname{InP}(111)$ A substrates. This system provides ample electron and hole confinement in the QDs, due to the strong GaAs bandgap reduction produced by its $3.8 \%$ tensile lattice mismatch with respect to InAlAs (Fig. 3(b)). ${ }^{18,22}$ Samples are grown in a VEECO Modular GEN II solid-source molecular beam epitaxy (MBE) system. To initiate growth, the native oxide is desorbed from the InP substrate at $485^{\circ} \mathrm{C}$ under an $\mathrm{As}_{4}$ flux. A $50 \mathrm{~nm} \mathrm{In}_{0.53} \mathrm{Ga}_{0.47}$ As buffer (InGaAs) is grown at $510^{\circ} \mathrm{C}$ on the $\operatorname{InP}(111) \mathrm{A}$, which promotes smooth growth of the subsequent $200 \mathrm{~nm}$ InAlAs layer at the same temperature. Growth parameters were investigated to optimize the surface smoothness for InGaAs and InAlAs on $\operatorname{InP}(111) A$ and will be reported elsewhere. ${ }^{23}$ For QD self-assembly, 0-5 monolayers (ML) of tensile strained GaAs is deposited at $485^{\circ} \mathrm{C}$ with a nominal $\mathrm{V} / \mathrm{III}$ ratio of 75 using a growth rate of $0.09 \mathrm{ML} / \mathrm{s}$. The GaAs layer is either left uncapped for observation by atomic force microscopy (AFM), or the GaAs is capped with $100 \mathrm{~nm}$ of InAlAs for PL and transmission electron microscopy (TEM) measurements. For the capped QD samples, the first $10 \mathrm{~nm}$ of InAlAs is grown at $485^{\circ} \mathrm{C}$ immediately after GaAs deposition, followed by growth at $510^{\circ} \mathrm{C}$ for the remainder of the InAlAs. TEM images were acquired using a FEI Osiris operating at $200 \mathrm{keV}$.

Both conventional PL and micro-photoluminescence ( $\mu \mathrm{PL}$ ) measurements were performed on the capped GaAs/ InAlAs(111)A samples. Each setup uses a frequency-doubled neodymium-YAG laser at $532 \mathrm{~nm}$. For conventional PL measurements, the PL signal was collected using an InGaAs detector via a standard lock-in technique. To address the emission of single QDs, high spectral resolution $(\mu \mathrm{PL})$ measurements were taken under continuous-wave excitation at cryogenic temperatures. The collected photons were analyzed by a 1500 lines $/ \mathrm{mm}$ grating spectrometer and a liquid nitrogen cooled Si-CCD with an overall resolution limit of $30 \mu \mathrm{eV}$. To enhance collection efficiency of the QD emissions, we increased the effective numerical aperture using a hyper-hemispheric solid immersion lens. FSS was determined by measuring the polarization dependence of the $\mu \mathrm{PL}$ spectra. For this purpose, a half-waveplate and a linear polarizer were introduced into the optical path. The linear polarizer was fixed to the high-reflectivity axis of the monochromator, and spectra were taken at $5^{\circ}$ increments of the half-waveplate.

Self-assembly of the tensile GaAs(111)A QDs is shown in Fig. 1. Prior to GaAs growth, the InAlAs(111)A surface morphology consists of gradual hills with monolayer-high contour lines. The InAlAs atoms arrange into layers of concentric 2D islands, forming structures that resemble "wedding cakes" (Fig. 1(a)). ${ }^{23}$ Similar wedding cake features form during MBE growth of certain other III-V materials including GaSb(001). ${ }^{24}$
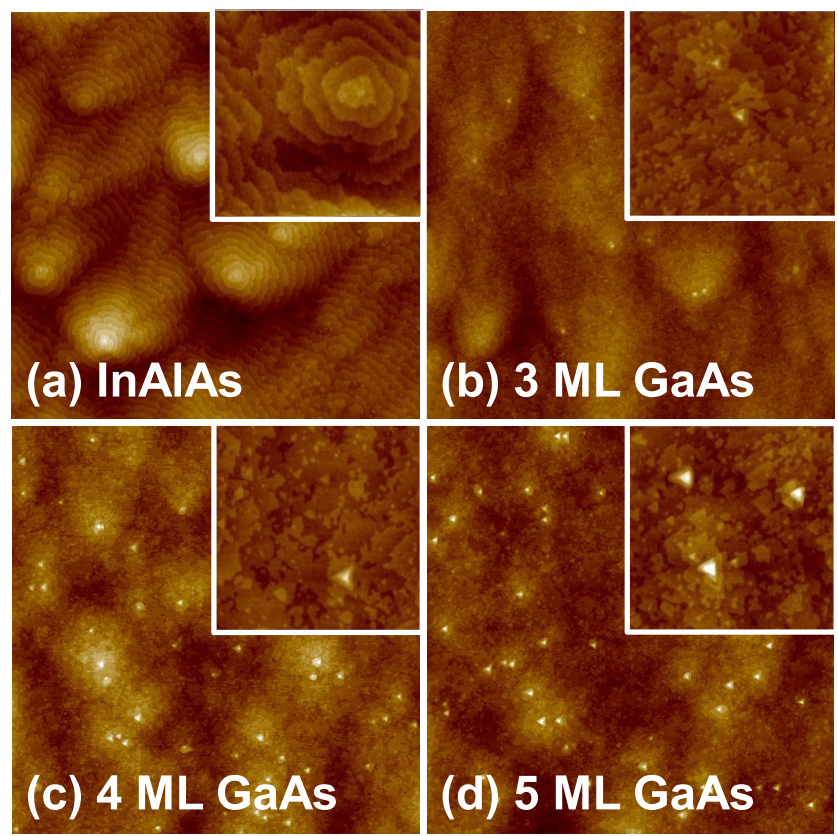

FIG. $1.5 \times 5 \mu \mathrm{m}^{2}$ AFM images of tensile GaAs self-assembly on InAlAs(111)A. The height scales are $5 \mathrm{~nm}$. (a) InAlAs prior to GaAs growth, (b) 3 ML GaAs, (c) 4 ML GaAs, and (d) 5 ML GaAs. Insets: $1 \times 1 \mu \mathrm{m}^{2} \mathrm{AFM}$ images with $3 \mathrm{~nm}$ height scales.

The InAlAs(111)A surface has an rms roughness of $0.41 \mathrm{~nm}$ averaged over a $5 \times 5 \mu \mathrm{m}^{2}$ area. GaAs deposited onto the InAlAs initially conforms to the 2D morphology for $<3 \mathrm{ML}$ of deposition (not shown). For $3 \mathrm{ML}$ of GaAs, 3D islands begin to nucleate at the apexes of the underlying InAlAs hills with a density of $1.0 \times 10^{8} \mathrm{~cm}^{-2}$ (Fig. 1(b)). By $4 \mathrm{ML}$ of deposition, 3D islands have formed throughout the surface with a density of $2.3 \times 10^{8} \mathrm{~cm}^{-2}$ (Fig. 1(c)). Increasing the GaAs thickness to $5 \mathrm{ML}$, the QD density remains constant, while their size increases (Fig. 1(d)). QD heights are measured with respect to the sample surface surrounding the QDs, and do not include the 2D GaAs layer beneath. Height distributions of $1.38 \pm 0.36 \mathrm{~nm}$ and $2.04 \pm 0.39 \mathrm{~nm}$ were measured for the $4 \mathrm{ML}$ and $5 \mathrm{ML}$ samples, respectively. The QD widths are $54 \pm 16 \mathrm{~nm}$ and $77 \pm 15 \mathrm{~nm}$, respectively, measured perpendicular to the edges of the triangular QDs. The successful formation of 3D islands under tensile strain contrasts starkly with previous attempts to grow (111) QDs under compressive strain, which only exhibit $2 \mathrm{D}$ growth.,

The very low QD densities obtained are desirable for spectroscopic studies of single QDs, ${ }^{25}$ but they also imply that little of the deposited GaAs incorporates into the 3D islands. From the QD size and density, the GaAs QDs only account for $0.14 \%$ and $0.30 \%$ of the deposited material by volume for the $4 \mathrm{ML}$ and $5 \mathrm{ML}$ samples, respectively. Therefore, the growth is primarily $2 \mathrm{D}$, with $3 \mathrm{D}$ islands forming concurrently after the deposition surpasses a critical thickness. This growth mode differs from conventional S-K growth, in which growth occurs primarily in the 3D islands after the 2D-to-3D transition. ${ }^{26}$ In our case, GaAs tends to incorporate at the many step edges on the InAlAs surface, contributing to $2 \mathrm{D}$ growth that changes the step edge morphology, as seen in Fig. 1 (see insets). This growth regime results in QDs with sufficiently low density ${ }^{25}$ to allow single QD measurements without substrate patterning. ${ }^{27}$ 

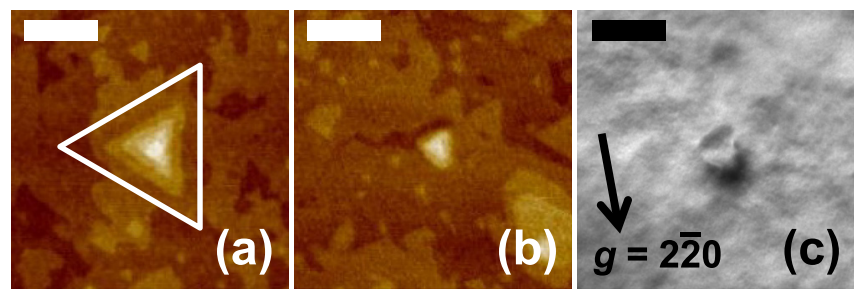

FIG. 2. QD morphology for $4 \mathrm{ML}$ GaAs samples. (a) AFM image of a large QD showing the symmetric shape. An equilateral triangle is drawn for comparison. (b) AFM image showing a QD of typical size. (c) Plan-view TEM image of a capped QD, acquired using a bright field, $g=2 \overline{2} 0$ two-beam condition. All scale bars are $100 \mathrm{~nm}$. AFM height scales are $3 \mathrm{~nm}$.

Tensile self-assembled growth represents a simple, robust approach for forming symmetric (111)A QDs. Consistent with the 3-fold symmetry of the (111)-surface, the QDs take the shape of triangular pyramids that are nearly equilateral about the base (Figs. 2(a) and 2(b)). The edges of the triangular base lie parallel to the in-plane $\langle 0 \overline{1} 1\rangle$ directions, parallel to the direction of atomic nearest neighbors for identical atoms (i.e., group-III or group-V). Both the highly symmetric QD shape and the [111]-oriented piezoelectric field provide the symmetric carrier confinement essential for low FSS, ${ }^{6,7}$ as discussed later in the $\mu \mathrm{PL}$ section. In contrast with other approaches to form (111) QDs, tensile selfassembly occurs during a single growth step, without the need for substrate pre-patterning to facilitate $3 \mathrm{D}$ growth. The strain-driven technique also offers the advantage of high temperature QD growth (observed from $460-540{ }^{\circ} \mathrm{C}$ in this study), which avoids the challenge of low temperature (e.g., $150-350{ }^{\circ} \mathrm{C}$ ) growth defects associated with droplet epitaxy. ${ }^{16}$

Plan-view TEM imaging reveals the morphology and crystalline quality of the capped QDs. Fig. 2(c) shows a representative QD in the $4 \mathrm{ML}$ GaAs sample. The QDs maintain their triangular shape after capping. By comparing the QD orientation in the TEM images to the selected area diffraction pattern (not shown), we have confirmed that the edges of the QDs lie parallel to $\langle 0 \overline{1} 1\rangle$, as seen by AFM (Figs. 2(a) and 2(b)). The lateral sizes of the capped QDs are $35-56 \mathrm{~nm}$ for the $4 \mathrm{ML}$ QDs and 36-67 nm for the 5 ML QDs. These values are smaller than the uncapped QDs observed by AFM, which we attribute to mass transport during the overgrowth of the QDs. ${ }^{28}$ For the $4 \mathrm{ML}$ sample, most of the tensile QDs observed have crescent-shaped strain contrast lobes, similar to TEM images of conventional QDs under compressive strain. ${ }^{29}$ For our GaAs(111)A QDs, the strain lobes and the absence of dislocations confirm that they are coherently strained to the InAlAs barriers. However, stacking faults (SFs) were occasionally observed in some QDs, suggesting that the threshold for plastic relaxation is close to $4 \mathrm{ML}$. For the $5 \mathrm{ML}$ sample, most of the QDs contained SFs or dislocations (not shown). Compared to our tensile GaAs QDs (e.g., Fig. 2(c)), attempts to grow compressive (111) QDs result in much higher densities of dislocations ${ }^{8}$ due to the lower nucleation barrier for dislocations under compressive strain. ${ }^{19}$ Further work is needed to fully suppress SFs in our tensile QDs, e.g., by adjusting the GaAs deposition thickness. The $4 \mathrm{ML}$ QD results shown here and tensile growth in other (111) material systems ${ }^{18,20}$ suggest that such defect-free growth is possible.

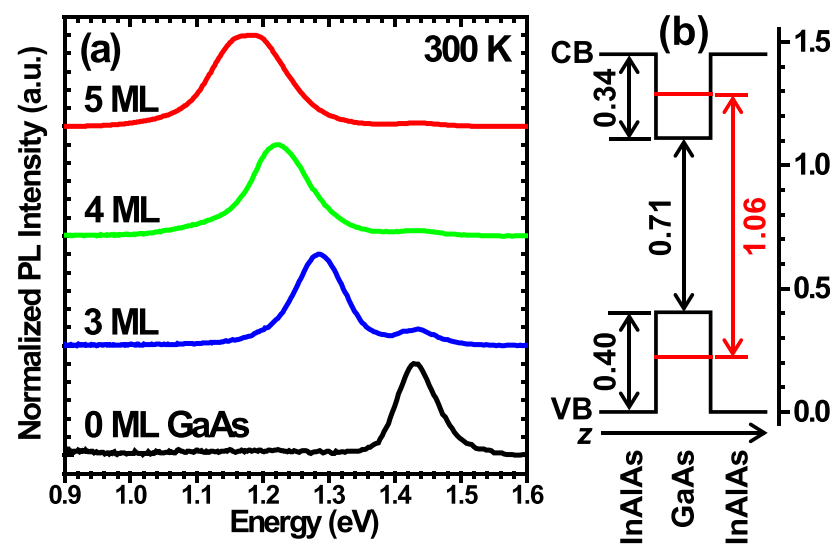

FIG. 3. (a) Room temperature PL of GaAs(111)A QDs with 0 ML, 3 ML, 4 $\mathrm{ML}$, and $5 \mathrm{ML}$ of GaAs. (b) Band diagram for GaAs(111)A QDs under $3.8 \%$ biaxial tension due to the lattice mismatch with $\operatorname{In}_{0.52} \mathrm{Al}_{0.48} \mathrm{As}$. The red value represents the calculated ground state emission for the $4 \mathrm{ML} \mathrm{GaAs}$ sample. All values are in $\mathrm{eV}$.

Each of the tensile GaAs/InAlAs(111)A samples exhibit PL at room temperature. Fig. 3(a) compares PL from the GaAs QD samples to an identical $0 \mathrm{ML}$ reference sample. Without GaAs present, the 0 ML sample shows only the InAlAs emission at $1.43 \mathrm{eV}$. For samples with 3-5 ML GaAs, strong PL peaks appear at lower energy due to the QDs. The QDs emit light from $1.18 \mathrm{eV}$ to $1.28 \mathrm{eV}$, reducing in photon energy with increasing GaAs thickness, in accordance with the quantum confinement effect. By reducing the measurement temperature, each GaAs PL spectrum can be resolved into QD ground states and higher energy transitions, including a wetting layer emission from the 2D GaAs layer (see supplemental material ${ }^{30}$ ). For each sample, PL from the GaAs QDs lies at a much lower energy than the bulk bandgap of GaAs $(1.42 \mathrm{eV})$. The reduction in PL energy is due to the $3.8 \%$ tensile lattice mismatch between GaAs and InAlAs, which strongly lowers the GaAs bandgap. ${ }^{18,22}$ Fig. 3(b) shows the band alignment in the GaAs/InAlAs(111)A QDs that results from the tensile strain. Also shown is an estimate of the ground state transition energy for the $4 \mathrm{ML}$ QDs, calculated with a particle-in-a-finite-box model. A rectangular-box model is used for simplicity, with QD dimensions taken from the AFM and TEM measurements. We define the out-of-plane confinement width of each QD as the sum of the QD height and the thickness of the 2D layer underneath. The approximate agreement between the estimated transition energy and the low energy side of the $4 \mathrm{ML}$ PL peak shows the prominent role of tensile strain in lowering the bandgap of the GaAs QDs. ${ }^{18,22}$

To measure the FSS of the tensile GaAs(111)A QDs, we performed high-resolution $\mu \mathrm{PL}$ of the $4 \mathrm{ML}$ QD sample at 9 K. Fig. 4(a) shows the $\mu$ PL spectrum of a single QD emission line, obtained without sample processing due to the low QD density. (See supplemental material for the complete $\mu \mathrm{PL}$ spectrum. ${ }^{30}$ ) This QD, emitting at $1.267 \mathrm{eV}$, exhibits a linewidth of $140 \mu \mathrm{eV}$, which suggests that the linewidths are broadened by spectral diffusion. ${ }^{31}$ Each QD line was fit to a Lorentzian profile to determine the QD peak position (Fig. 4(a)). Next, the peak position was measured as a function of the polarization angle, and the FSS was determined from the amplitude of a sinusoidal fit to the data (Fig. 4(b)). This 

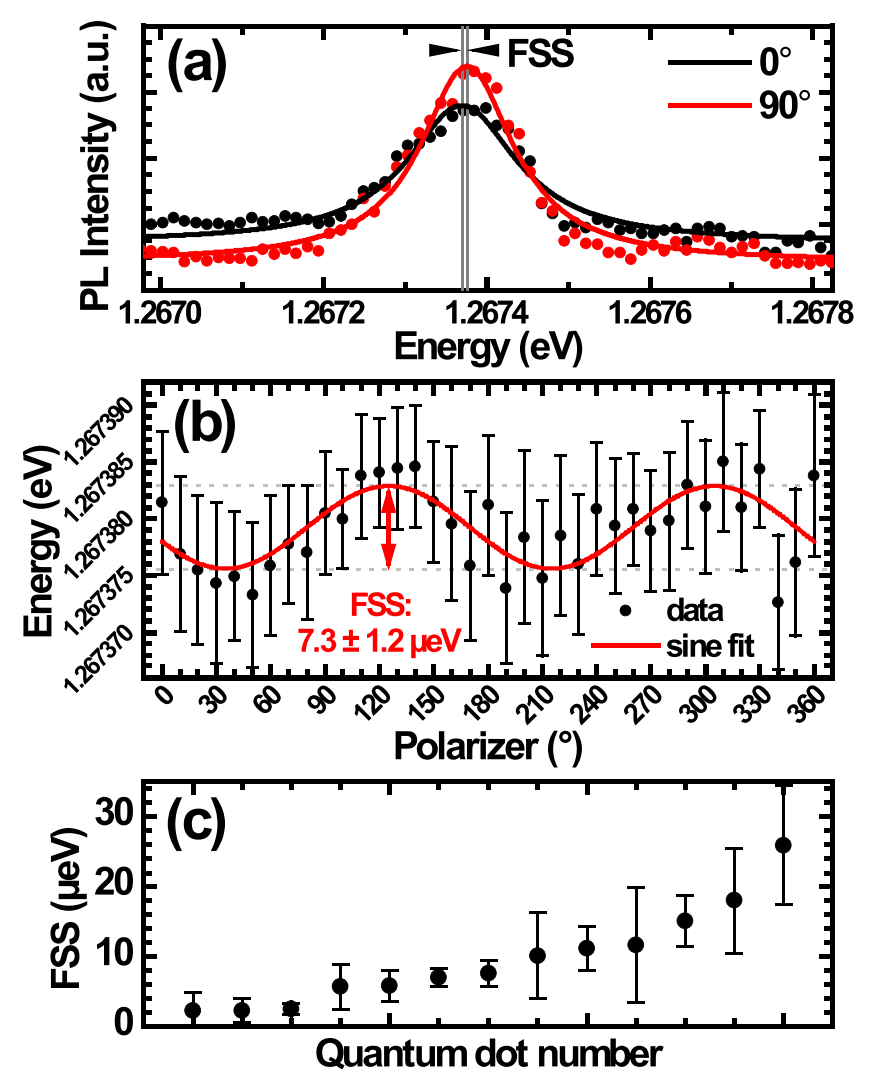

FIG. 4. (a) Polarization-resolved $\mu \mathrm{PL}$ of a 4 ML GaAs(111)A QD acquired at $9 \mathrm{~K}$. Data are fit to Lorentzian profiles. The peak position shifts with polarization direction due to FSS. (b) Peak position of the QD emission line shown in (a) versus the polarizer angle. A sinusoidal fit reveals an FSS value of $7.3 \pm 1.2 \mu \mathrm{eV}$. A linear energy shift caused by temperature drift during data acquisition $(\sim 0.3 \mathrm{~K})$ has been subtracted from the data. (c) FSS values of emission lines measured from 13 individual QDs in the $4 \mathrm{ML}$ GaAs sample.

procedure allows us to determine FSS down to $\sim 3 \mu \mathrm{eV}$. An FSS value of $7.3 \pm 1.2 \mu \mathrm{eV}$ was thereby measured for the QD studied in Figs. 4(a) and 4(b). We repeated this procedure for a number of QD lines, identifying 13 QD emissions with measurable FSS values, shown in Fig. 4(c). QD emissions with FSS below our detection limit were discarded from analysis, to avoid the possibility of including charged excitons that have inherently zero FSS. Thus, we have obtained a median FSS value of $7.6 \mu \mathrm{eV}$ for the $4 \mathrm{ML}$ GaAs(111)A QDs. The FSS distribution in Fig. 4(c) is well below the typical FSS of S-K-grown (001) QDs and is comparable to droplet epitaxy-grown (111) QDs. ${ }^{4,10-12}$ The low FSS values observed are consistent with high electronic symmetry in the (111) QDs due to the out-of-plane piezoelectric field, combined with the symmetric QD morphology that results from tensile self-assembly. ${ }^{6,7}$ Further reduction in FSS for the tensile (111) QDs is anticipated with optimization of growth conditions for the GaAs/InAlAs(111)A structure.

We have demonstrated the growth of high-symmetry GaAs(111)A QDs with low FSS using tensile-strained selfassembly. This simple technique forms QDs in a single growth step without low temperature or substrate patterning requirements. AFM and TEM measurements show that a low density of triangular QDs self-assemble from tensile GaAs grown on InAlAs(111)A. These results contrast with previous attempts to grow (111) QDs under compressive strain, where 3D island growth was not observed. ${ }^{8,9}$ Coherent quantum dots form for GaAs thicknesses up to $4 \mathrm{ML}$, similar to previous observations of dislocation-free tensile selfassembly on (111) surfaces. $^{18,20,21}$ The (111)A QDs emit strong room temperature PL for all samples in this study. Low-temperature $\mu \mathrm{PL}$ of the $4 \mathrm{ML}$ QDs shows single QD emission lines with a median FSS of $7.6 \mu \mathrm{eV}$, consistent with the low FSS predicted for (111) QDs. ${ }^{6,7}$ Tensile selfassembly thus represents a simple and robust technique to form (111) QDs with high structural and electronic symmetry, as desired for generating entangled photon pairs.

Microscopy facilities used in this work were supported by the Yale Institute for Nanoscience and Quantum Engineering and National Science Foundation MRSEC DMR 1119826. C.D.Y. acknowledges support from the Department of Energy Office of Science Graduate Fellowship Program (DOE SCGF), made possible in part by the American Recovery and Reinvestment Act of 2009, administered by ORISE-ORAU under Contract No. DE-AC05-06OR23100. Additional support was provided by the University of California Lab Fees Research Program (Grant No. 12-LR238568).

${ }^{1}$ A. J. Shields, Nat. Photonics 1, 215 (2007).

${ }^{2}$ O. Benson, C. Santori, M. Pelton, and Y. Yamamoto, Phys. Rev. Lett. 84, 2513 (2000).

${ }^{3}$ N. Akopian, N. H. Lindner, E. Poem, Y. Berlatzky, J. Avron, D. Gershoni, B. D. Gerardot, and P. M. Petroff, Phys. Rev. Lett. 96, 130501 (2006).

${ }^{4}$ R. Seguin, A. Schliwa, S. Rodt, K. Potschke, U. W. Pohl, and D. Bimberg, Phys. Rev. Lett. 95, 257402 (2005).

${ }^{5}$ R. M. Stevenson, R. J. Young, P. Atkinson, K. Cooper, D. A. Ritchie, and A. J. Shields, Nature 439, 179 (2006).

${ }^{6}$ A. Schliwa, M. Winkelnkemper, A. Lochmann, E. Stock, and D. Bimberg, Phys. Rev. B 80, 161307(R) (2009).

${ }^{7}$ R. Singh and G. Bester, Phys. Rev. Lett. 103, 063601 (2009).

${ }^{8}$ H. Yamaguchi, J. G. Belk, X. M. Zhang, J. L. Sudijono, M. R. Fahy, T. S. Jones, D. W. Pashley, and B. A. Joyce, Phys. Rev. B 55, 1337 (1997).

${ }^{9}$ H. Wen, Z. M. Wang, J. L. Shultz, B. L. Liang, and G. J. Salamo, Phys. Rev. B 70, 205307 (2004).

${ }^{10}$ J. Treu, C. Schneider, A. Huggenberger, T. Braun, S. Reitzenstein, S. Höfling, and M. Kamp, Appl. Phys. Lett. 101, 022102 (2012).

${ }^{11}$ T. Mano, M. Abbarchi, T. Kuroda, B. McSkimming, A. Ohtake, K. Mitsuishi, and K. Sakoda, Appl. Phys. Express 3, 065203 (2010).

${ }^{12}$ E. Stock, T. Warming, I. Ostapenko, S. Rodt, A. Schliwa, J. A. Tofflinger, A. Lochmann, A. I. Toropov, S. A. Moshchenko, D. V. Dmitriev, V. A. Haisler, and D. Bimberg, Appl. Phys. Lett. 96, 093112 (2010).

${ }^{13}$ G. Juska, V. Dimastrodonato, L. O. Mereni, A. Gocalinska, and E. Pelucchi, Nat. Photonics 7, 527 (2013).

${ }^{14}$ M. Felici, P. Gallo, A. Mohan, B. Dwir, A. Rudra, and E. Kapon, Small 5, 938 (2009).

${ }^{15}$ K. Watanabe, N. Koguchi, and Y. Gotoh, Jpn. J. Appl. Phys. 39, L79 (2000).

${ }^{16}$ K. Watanabe, S. Tsukamoto, Y. Gotoh, and N. Koguchi, J. Cryst. Growth 227, 1073 (2001).

${ }^{17}$ A. Dousse, J. Suffczynski, A. Beveratos, O. Krebs, A. Lemaitre, I. Sagnes, J. Bloch, P. Voisin, and P. Senellart, Nature 466, 217 (2010).

${ }^{18}$ C. D. Yerino, P. J. Simmonds, B. L. Liang, V. G. Dorogan, M. E. Ware, Y. I. Mazur, D. Jung, D. L. Huffaker, G. J. Salamo, and M. L. Lee, Appl. Phys. Lett. 105, 071912 (2014).

${ }^{19}$ P. J. Simmonds and M. L. Lee, J. Appl. Phys. 112, 054313 (2012).

${ }^{20}$ P. J. Simmonds and M. L. Lee, Appl. Phys. Lett. 99, 123111 (2011).

${ }^{21}$ A. Raviswaran, C. P. Liu, J. Kim, D. G. Cahill, and J. M. Gibson, Phys. Rev. B 63, 125314 (2001). 
${ }^{22}$ P. J. Simmonds, C. D. Yerino, M. Sun, B. Liang, D. L. Huffaker, V. G. Dorogan, Y. Mazur, G. Salamo, and M. L. Lee, ACS Nano 7, 5017 (2013).

${ }^{23}$ C. D. Yerino, P. J. Simmonds, B. L. Liang, D. L. Huffaker, and M. L. Lee, "Molecular beam epitaxy growth optimization of InAlAs and InGaAs alloys on InP (111)A, (111)B, and (110)" (unpublished).

${ }^{24}$ B. Z. Nosho, B. R. Bennett, E. H. Aifer, and M. Goldenberg, J. Cryst. Growth 236, 155 (2002).

${ }^{25}$ M. B. Ward, O. Z. Karimov, D. C. Unitt, Z. L. Yuan, P. See, D. G. Gevaux, A. J. Shields, P. Atkinson, and D. A. Ritchie, Appl. Phys. Lett. 86, 201111 (2005).
${ }^{26}$ D. Leonard, K. Pond, and P. M. Petroff, Phys. Rev. B 50, 11687 (1994).

${ }^{27}$ C. Santori, M. Pelton, G. Solomon, Y. Dale, and E. Yamamoto, Phys. Rev. Lett. 86, 1502 (2001).

${ }^{28}$ K. Yamaguchi, Y. Saito, and R. Ohtsubo, Appl. Surf. Sci. 190, 212 (2002).

${ }^{29}$ D. Leonard, M. Krishnamurthy, C. M. Reaves, S. P. Denbaars, and P. M. Petroff, Appl. Phys. Lett. 63, 3203 (1993).

${ }^{30}$ See supplemental material at http://dx.doi.org/10.1063/1.4904944 for low temperature PL spectra and additional $\mu \mathrm{PL}$ spectra.

${ }^{31}$ A. V. Uskov, I. Magnusdottir, B. Tromborg, J. Mork, and R. Lang, Appl. Phys. Lett. 79, 1679 (2001). 